\title{
Engajamento Nutricional
}

- Atualmente, as doenças crônicas não transmissíveis (DCNT), principalmente doenças cardiovasculares, câncer, diabetes e doenças respiratórias crônicas são a maior causa de mortes no mundo e mortes prematuras em países de baixa e média renda, como o Brasil;

- A maioria dessas mortes pode ser amplamente evitada por meio da mudança de estilo de vida e de fatores de risco, como o tabagismo, consumo de álcool, hábitos alimentares, obesidade e atividade física;

- Considerando a crescente morbidade e mortalidade decorrente de DCNT junto à dificuldade das pessoas em engajar nas mudanças, uma das principais prioridades deve ser o desenvolvimento de iniciativas que apoiem o autogerenciamento, a modificação do estilo de vida e a adesão às mudanças;

- Engajamento significa empenho, envolvimento, participação ativa e compromisso dos indivíduos frente ao seu estilo de vida, mas para que isso aconteça é preciso entender que estamos diante de seres biopsicossociais, sendo que uma série de determinantes influenciam suas respostas biológicas e comportamentais;

- Estudos apontam que tanto fatores ambientais, como variedade, qualidade e propaganda de alimentos, quanto fatores pessoais, como estágio de prontidão para mudança, auto eficácia e percepção dos prós e contras da mudança, afetam o comportamento alimentar, sendo que os últimos são preditores mais fortes das escolhas e ingesta alimentar;

- Técnicas de mudança de comportamento que apoiam a mudança de hábitos alimentares em curto e longo prazo envolvem o estabelecimento de metas, auto monitoramento, tarefas graduais e feedback, entre outras abordagens;

- Facilitar o processo de mudança, melhorar a eficácia e o engajamento das pessoas também requer o desenvolvimento de habilidades de comunicação e relacionamento por parte dos profissionais de saúde, como a empatia, escuta, presença e reflexão;

- Compreendendo a complexidade do ser humano e a situação única de cada indivíduo é possível atuar como um agente de mudanças, criando um ambiente colaborativo e de parceria, reduzindo a resistência e ambivalência, aumentando a consciência, eficácia e recursos para a mudança, tornando o indivíduo um protagonista do processo de construção de novos hábitos!

\section{Referências bibliográficas}

1. KHAN, N. et al. Digital Health Technologies to Promote Lifestyle Change and Adherence. Curr Treat Options Cardiovasc Med;19(8):60, 2017.

2. SAMDAL, G.B. et al. Effective behaviour change techniques for physical activity and healthy eating in overweight and obese adults; systematic review and meta-regression analyses. Int J Behav Nutr Phys Act;14(1):42, 2017.

3. CHRISTIE, D.; CHANNON, S. The potential for motivational interviewing to improve outcomes in the management of diabetes and obesity in paediatric and adult populations: a clinical review. Diabetes Obes Metab;16(5):381-7, 2013.

4. OLVERA ALVAREZ, H.A. et al. An Integrated Socio-Environmental Model of Health and Well-Being: a Conceptual Framework Exploring the Joint Contribution of Environmental and Social Exposures to Health and Disease Over the Life Span. Curr Environ Health Rep; 5(2):233-243, 2018.

5. MENEZES, M. et al. Individual and food environmental factors: Association with diet. Public Health Nutrition; 21(15), $2782-2792,2018$. 